\title{
Priorização na Resolução de Manifestações Patológicas em Estruturas de Concreto Armado: Método GUT
}

GUT method employed on the prioritization of resolution of pathologic manifestations in reinforced concrete structures

\author{
Natália Martins ${ }^{1}$ (id) orcid.org/0000-0001-5648-6765 \\ Raniely Pessoa ${ }^{2,3}$ (id) orcid.org/0000-0003-4321-1513 \\ Rayssa Nacimento 4 (iD orcid.org/0000-0002-0012-9643 \\ ${ }^{1}$ Departamento de Ciência e Engenharia de Materiais, Universidade Federal da Paraíba, João Pessoa, Paraíba, Brasil, \\ 2 Departamento de Engenharia Civil, Faculdade Maurício de Nassau, João Pessoa, Paraíba, Brasil, \\ 3 Departamento de Engenharia Civil, Faculdade Internacional da Paraíba, João Pessoa, Paraíba, Brasil, \\ ${ }^{4}$ Departamento de Engenharia Civil, Centro Universitário e João Pessoa, João Pessoa, Paraíba, Brasil. \\ E-mail do autor principal: Natália Martins, nataliapiresmartins@hotmail.com
}

\section{Resumo}

Planejar a aplicação das correções das manifestações patológicas em concreto armado é um ponto relevante na redução dos custos associados ao problema. Desse modo, vem à tona a possibilidade de emprego de métodos com o objetivo de relacionar as anomalias à sua prioridade de resolução. Este trabalho demonstrou o emprego da matriz de priorização GUT com esse objetivo particular. Foram apresentados registros fotográficos dos principais problemas encontrados em uma área de estudo, nos quais aplicou-se o método. Os resultados indicaram que as correções devem priorizar elementos estruturais que apresentam corrosão de armaduras. Concluiu-se que o método GUT representou uma ferramenta útil neste ramo da construção civil. Além disso, seus resultados servirão para beneficiar a empresa ou instituição através da retroalimentação do sistema, com informações acerca das principais causas e origens dos problemas, possibilitando a realização de melhoramentos para prevenção da ocorrência de manifestações.

Palavras-Chave: Matriz de Priorização; Método GUT; Patologia das construções; Concreto Armado.

\begin{abstract}
Planning the resolution of pathological manifestations in reinforced concrete structures is a relevant point in the reduction of costs. This brings to the fore the possibility of employing methods with the objective of relating the problems to their resolution priority. This work demonstrated the use of the GUT prioritization matrix with this particular purpose. Photographic records of the main problems found in a study area, in which the method was employed, were presented. Results show that corrosion should be corrected first. It was concluded that the GUT method was a useful tool to prioritize pathological manifestations in an area where corrections are required. In addition, its results
\end{abstract} 139

http: / / dx.doi.org/10.25286/repa.v2i3.707 
can benefit the company or institution through feedback of the system with information about the main causes and origins of the problems, indicating improvements to avoid the occurrence of pathological manifestations.

Key-words: Prioritization Matrix; GUT Method; Building Pathology; Reinforced Concrete.

\section{Introdução}

Grande parte das patologias nas edificações apresenta manifestações características que possibilitam a identificação da sua origem, bem como suas possíveis consequências. No que diz respeito às estruturas de concreto armado, a degradação é um problema que atinge a construção civil em todo o mundo, principalmente devido à ocorrência precoce. Thomaz avalia que a evolução tecnológica induziu à execução de obras em curto prazo, fazendo o uso de estruturas mais leves e exigências de baixo custo [1]. A estes fatores, soma-se o despreparo dos profissionais, o que leva a obtenção de produtos finais de baixa qualidade. A falta de planejamento e de fiscalização contribui de forma expressiva para o agravamento do quadro.

Ainda de acordo com Thomaz, as manifestações patológicas são evolutivas e tendem a acentuar-se com o tempo, podendo gerar o aparecimento de novos problemas em decorrência dos já existentes [1]. Nesse sentido, percebe-se a importância do diagnóstico precoce na previsão das consequências futuras para o desempenho da edificação; quanto mais cedo sua realização, mais duráveis, efetivas e fáceis serão as correções. No que se refere aos custos da correção, Meira avalia que estes podem ser bastante significativos e que aumentam à medida que a intervenção se torna mais tardia [2].

Uma questão importante, levantada por Guimarães et al., trata da resolução parcial do problema quando não há recursos suficientes para que seja realizada manutenção em todas as estruturas que apresentem manifestações patológicas [3]. Sabe-se que um grande número de empresas e órgãos públicos encontram dificuldades em alocar seus limitados recursos em reformas e manutenção de suas edificações. Estas dificuldades estão ligadas ao grande número de edificações, de manifestações patológicas e à falta 140 de uma metodologia que possa ser aplicada à priorização da aplicação [3].

Desse modo, além do fator custo, o emprego de ferramentas eficazes em estabelecer uma relação de priorização na resolução de problemas é identificado como um aspecto relevante na correção e prevenção de manifestações patológicas. Para este fim, este artigo demonstra que podem ser utilizados métodos comumente empregados para gestão estratégica de negócios, no âmbito da Administração de Empresas e da Engenharia de Produção.

O principal objetivo deste trabalho é atestar a eficácia do emprego método GUT na priorização da resolução de manifestações patológicas em estruturas de concreto armado, facilitando a alocação de recursos de empresas e instituições públicas destinados à manutenção de suas edificações.

\section{Manifestações patológicas em concreto armado}

Os problemas patológicos podem ser originados nas etapas de projeto, de escolha de materiais, de execução da construção e de uso e manutenção. E podem ser causados por ações mecânicas, físicas, químicas e biológicas [4].

Silva destaca como ações mecânicas a erosão e o excesso de cargas não previstas em projeto, provocando fissuras e, consequentemente, possibilitando a instalação de novos problemas [5].

Souza e Ripper destacam como ações físicas as variações de temperatura, pois promovem os diferentes comportamentos relacionados à dilatação térmica dos materiais, devido aos seus diferentes coeficientes de dilatação [6]. 
Já as ações químicas são as principais causadoras de patologias, pois o concreto, normalmente, tem uma resistência baixa a esse tipo de ataque. As substâncias encontradas no meio ambiente penetram no material e, sob determinadas condições de temperatura e umidade, provocam reações nocivas ao concreto [7].

Segundo Silva, as ações químicas mais comuns são [5]:

- reações álcali-agregado, que resultam num gel higroscópico expansivo, causando fissuras, expansões, redução da resistência à tração e à compressão e exsudação de gel;

- ataque por cloretos, que, segundo Figueiredo, podem ser introduzidos no concreto por diversas formas, como: impurezas já presentes nos constituintes do concreto, através de aditivos aceleradores de pega, através da água salgada e maresias (em caso de ambientes marinhos) e em etapas de processo industrial [8];

- ataque por sulfatos, que, de acordo com Brandão, são elementos muito agressivos, causam fissuração no concreto e desprendimento de lascas [7];

- carbonatação, reação entre os compostos hidratados do cimento e o $\mathrm{CO} 2$, que provoca uma redução do $\mathrm{pH}$ capaz de desestabilizar a camada passivante das armadura, podendo iniciar um processo de corrosão generalizada [9].

De acordo com a ABNT NBR: 6118 de 2014 [10], o ataque por íons cloretos e a carbonatação são os principais agentes despassivadores que levam à corrosão de armadura.

Com relação às ações biológicas, Silva cita como alguns agentes causadores da deterioração da estrutura, o desenvolvimento de organismos e micro-organismos e o crescimento da vegetação na estrutura [5].

Em um estudo feito nas edificações do Estado do Ceará, Silva concluiu que a manifestação predominante foi a corrosão de armaduras, com um índice de $96,67 \%$ dos casos, como mostra a Figura 1 [5].

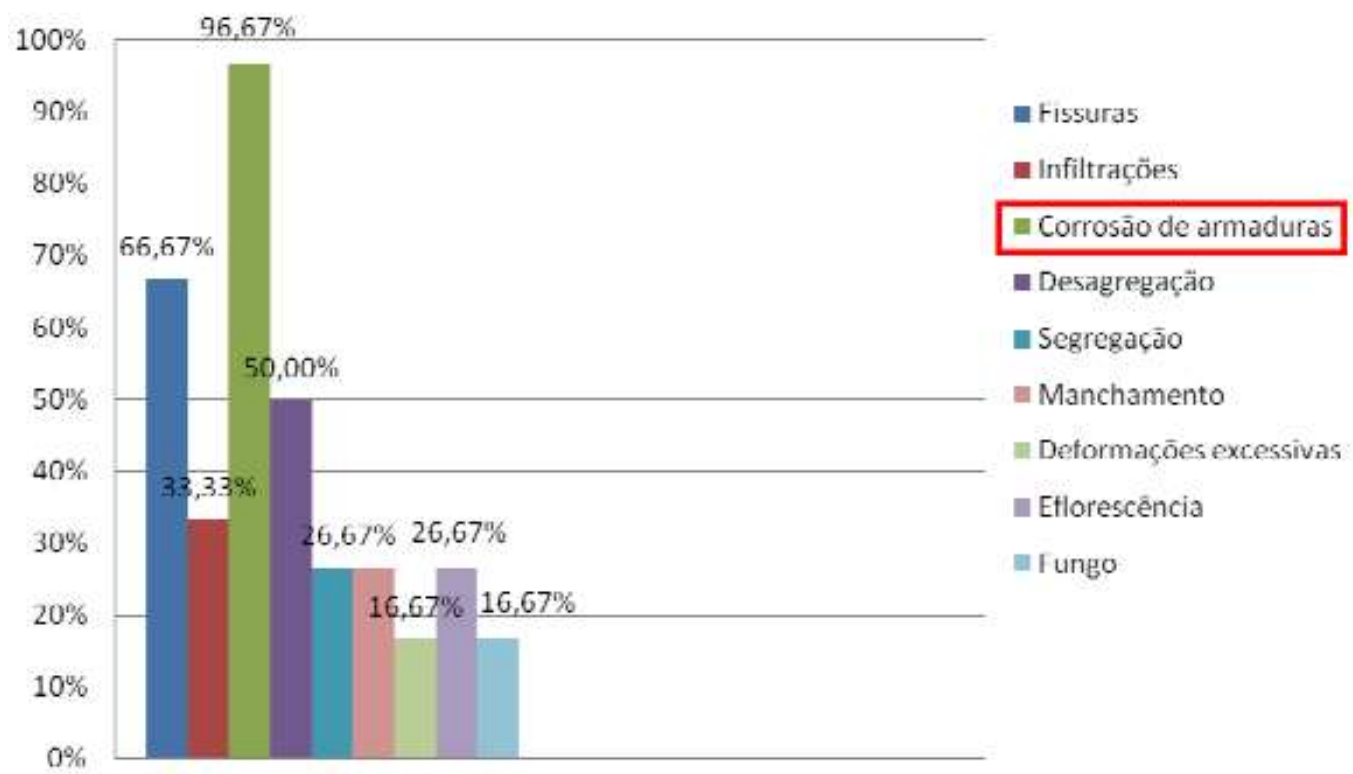

Figura 1: Incidência das manifestações patológicas nas estruturas de concreto (CE). Fonte: Silva (2011).

Esse diagnóstico reflete resultados de outros estudos voltados para degradação da estrutura de concreto realizados em outras regiões do país, 
destacando-se os trabalhos de Aranha, que observou que $46 \%$ das manifestações patológicas na região Norte era de corrosão de armadura [11]; Andrade chegou ao número de $64 \%$ em Recife [12]; Nince, na região Centro-Oeste, valores de $30 \%$ [13].

\section{Ferramentas de gerenciamento de risco}

Lichtenstein propôs um método genérico para ser empregado na Patologia das construções. A primeira fase consiste no levantamento de subsídios necessários ao entendimento dos fenômenos, através de vistoria do local, anamnese, ensaios complementares e pesquisa. A segunda etapa é a elaboração do diagnóstico, com a identificação de causa e efeitos do problema patológico. O terceiro passo é a definição da conduta para resolver o problema, tendo sido levantadas as hipóteses de evolução futura e as alternativas de intervenção. Ao fim, deve ser feito o registro do caso, com a finalidade de guardar 0 histórico de intervenções e retroalimentar o processo [14].

Deve-se levar em conta que o diagnóstico é uma etapa que possui forte dependência da experiência dos avaliadores e leva em conta seu conhecimento prévio sobre manifestações patológicas em concreto armado. Desse modo, trata-se de uma etapa que está intimamente ligada a fatores subjetivos, como a sensibilidade e a competência do profissional avaliador.

A definição das terapêuticas a serem aplicadas a fim de atacar as raízes dos problemas deve ser realizada com cautela, pois sua eficiência está diretamente ligada à prevenção da reincidência de patologias. Só então deve-se proceder o serviço de manutenção.

O procedimento proposto por Lichtenstein [14] é aplicado diretamente quando a aplicação das terapêuticas corretivas não requer estabelecimento de uma hierarquia do nível de risco. No entanto, em situações de escassez de recursos financeiros, o planejamento da execução de manutenção pode se basear na priorização dos casos.

Alguns autores brasileiros vêm realizando 142

estudos sobre o emprego de ferramentas de gerenciamento de risco aplicadas à hierarquização de níveis de degradação em inspeções prediais (instalações hidráulicas e sanitárias [15], alvenaria [3], fachadas [16] e estruturas de concreto armado [17]). Essas ferramentas podem ser empregadas individualmente ou combinadas, proporcionando resultados que permitem análises mais completas.

O Instituto Brasileiro de Avaliações e Perícias de Engenharia [18] recomenda a utilização dos métodos GUT (Gravidade, Urgência e Tendência) ou FMEA: (Failure Mode and Effect Analisys ou, em português, análise dos Modos de Falhas e seus Efeitos), ferramentas de Gestão da Qualidade, para estabelecer a ordem de prioridades na aplicação das terapias de correção. A prioridade é disposta em ordem decrescente quanto ao grau de risco e intensidade das anomalias e falhas.

Silva e Monteiro empregaram duas ferramentas de gestão da qualidade combinadas, análise da árvore de falhas (FTA) e a análise dos Modos de Falhas e seus Efeitos (FMEA), na detecção e controle de patologias e estruturas de concreto armado do Aeroporto Internacional de Recife-PE [17].

Uma outra metodologia foi elaborada por Guimarães et al., que realizou um estudo em prédios da Universidade Federal de Goiás (UFG). Em seus estudos, os autores estabeleceram uma ordem de grau de deterioração para definir um programa de manutenção através de uma avaliação comparativa do grau de deterioração de edificações de características similares ou não [3]. Esse método foi utilizado com êxito por Pacheco e Vieira em inspeção de fachadas de edifícios [16].

Em todos os estudos citados, é recomendado que o emprego dos métodos seja complementado com a proposição de planos de ação corretiva e preventiva para as manifestações identificadas, isto é, a execução da terceira etapa do procedimento genérico elaborado por Lichtenstein [14], bem como o registro e a retroalimentação do processo.

\subsection{Método GUT}


O método GUT (Gravidade, Urgência e Tendência) é uma ferramenta desenvolvida por Charles H. Kepner e Benjamin B. Tregoe para definir prioridades de forma racional [19]. Consiste em analisar a gravidade ou o impacto do problema nas operações e pessoas envolvidas, a urgência ou a brevidade necessária para a resolução dos problemas e a tendência ou apresentação de melhora ou piora do problema. Ele se utiliza de três escalas (colunas de decisão) para definir uma prioridade, e através da combinação delas é possível fazer uma priorização eficaz, orientando a tomada de decisão e a resolução de problemas. A Tabela 1 apresenta as escalas empregadas pelo método [20].
É necessário que cada problema a ser analisado receba uma nota de 1 a 5 em cada uma das características: gravidade, urgência e tendência. Os pontos da escala GUT atribuídos a cada problema são multiplicados, dando origem a um valor resultante para cada problema. Desta forma, as ações de gerenciamento podem ser pautadas segundo os valores máximos obtidos.

Tabela 1: Pontuação GUT.

\begin{tabular}{|c|c|c|c|}
\hline Pontos & Gravidade & Urgência & Tendência \\
\hline & $\begin{array}{l}\text { Consequência se nada } \\
\text { for feito }\end{array}$ & $\begin{array}{l}\text { Prazo para tomada } \\
\text { de decisão }\end{array}$ & $\begin{array}{c}\text { Proporção do problema } \\
\text { no futuro }\end{array}$ \\
\hline 5 & $\begin{array}{l}\text { Prejuízos extremamente } \\
\text { graves }\end{array}$ & É necessário ação imediata & Se nada for feito, agravamento imediato \\
\hline 4 & Muito graves & Com alguma urgência & Vai piorar a curto prazo \\
\hline 3 & Graves & O mais cedo possível & Vai piorar a médio prazo \\
\hline 2 & Pouco graves & Pode esperar um pouco & Vai piorar a longo prazo \\
\hline 1 & Sem gravidade & Não tem pressa & Não vai piorar \\
\hline
\end{tabular}

Fonte: OLIVEIRA (1995).

\section{Metodologia}

Para realizar a priorização das manifestações patológicas, foi empregado o método GUT nas estruturas de concreto armado do Centro de Tecnologia do Campus I da Universidade Federal da Paraíba. A área de estudo conta com edificações de diferentes usos: salas de aula, ambientes de professores, administração, laboratórios e auditórios. Os prédios possuem variadas idades e são, no geral, diferentes (exceto os blocos A, B, C, $D$ e $E$, onde funcionam salas de aula $e$ laboratórios).

Foram visitadas 18 edificações. Os elementos estruturais, lajes, vigas e pilares, foram analisados individualmente. Foi realizado o registro fotográfico das anomalias identificadas com o objetivo de facilitar o diagnóstico e criar um banco de dados.

Os levantamentos necessários à elaboração do cadastro de problemas e manifestações patológicas foram executados através de inspeção visual, durante visitas ao local.

Através da formulação das hipóteses sobre as causas dos problemas encontrados nas visitas, pôde-se buscar evidências que comprovassem essas hipóteses para realizar o diagnóstico. Desse modo, procedeu-se o registro dos diagnósticos e a respectiva verificação da frequência e do grau de deterioração existente.

Anteriormente à aplicação do método GUT dos dados, procedeu-se o tratamento das informações coletadas. Esse tratamento prévio se deu através da divisão das manifestações patológicas em grupos, quais sejam: corrosão de armaduras, segregação, desagregação, manchas,

http: / / dx.doi.org/10.25286/repa.v2i3.707 
eflorescência e fissuras. Cada grupo representa o tipo de manifestação patológica observada nas visitas. Uma vez que as mesmas se apresentam sob variadas urgências, gravidades e tendências, procedeu-se uma divisão em subgrupos, de acordo com o nível de degradação. Por fim, as anomalias observadas foram catalogadas de acordo com os subgrupos definidos, nos quais foi atribuída, pelos avaliadores, uma pontuação GUT. Este método de aplicação da matriz GUT baseou-se no empregado por Knapp e Olivan para priorizar anomalias observadas em estruturas metálicas, tendo sido aplicado de forma mais simplificada e direta, com algumas alterações [21].

Além da pontuação GUT, foi atribuída a cada categoria um valor que representa a incidência de anomalias, isto é, a quantidade de vezes que o problema se manifestou na área de estudo.

\section{Resultados}

Algumas das manifestações patológicas encontradas estão apresentadas nas Figuras 2 e 3.

Na Figura 2, nota-se a presença de segregação cuja causa provável é o lançamento do concreto na fôrma de altura superior à recomendada. A prevenção dessa manifestação patológica pode ser feita, portanto, na etapa de execução da obra.

A Figura 3 apresenta uma laje de concreto armado com armadura exposta em processo de corrosão, além de significativa desagregação de concreto. Esse caso particular está associado a uma reforma não planejada que incluiu a instalação de tubulações sanitárias através do elemento estrutural. A imagem sugere que a execução não se deu de forma adequada, gerando o surgimento de uma série de anomalias na laje.

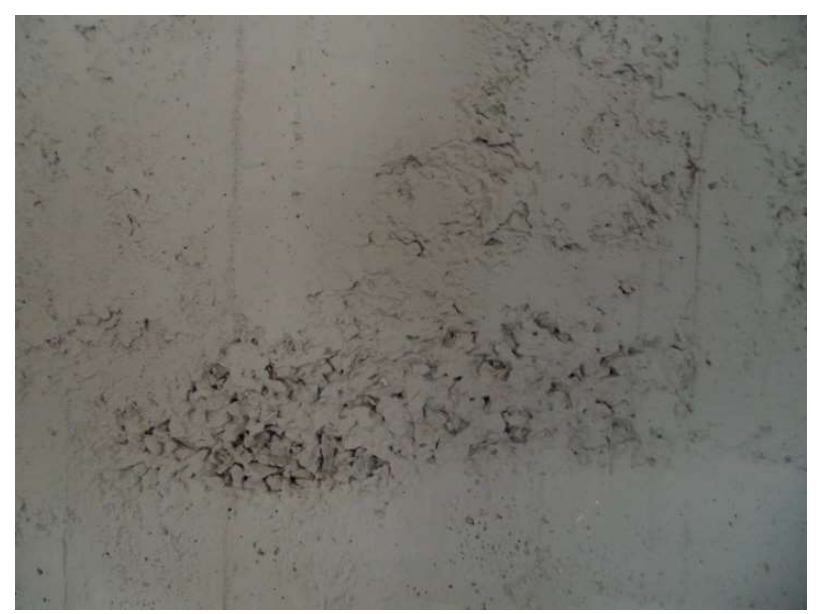

Figura 2: Segregação em parede de concreto armado. Fonte: Autores (2017). 


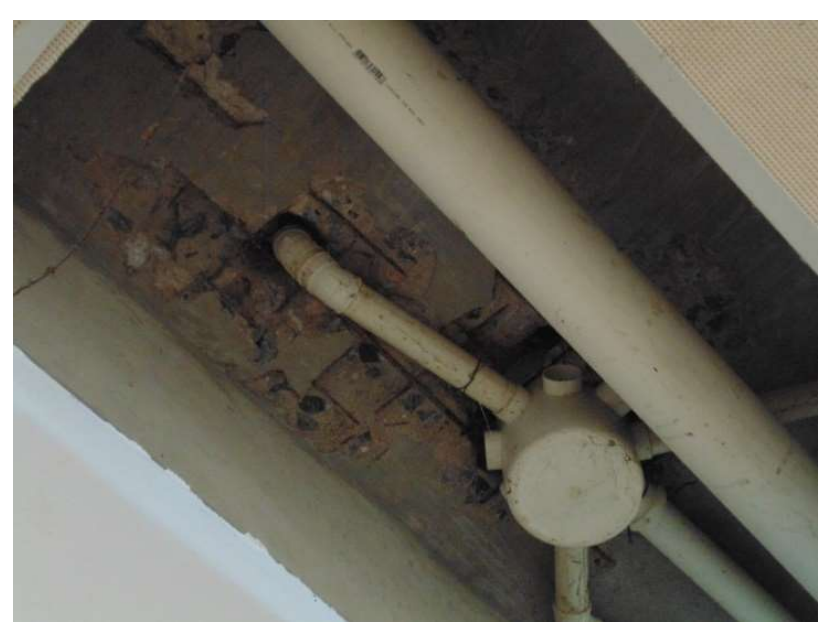

Figura 3: Corrosão de armadura e desagregação em laje de concreto armado. Fonte: Autores (2017).

Alguns dos prédios já haviam passado por manutenção anterior em sua estrutura, de acordo com o que foi observado. Diversos elementos estruturais haviam passado por terapêuticas corretivas, como preenchimento de trincas e recomposição do concreto. No entanto, notou-se a reincidência de manifestações patológicas mesmo após os reparos. Nesse ponto, ressalta-se a importância de adotar a terapêutica adequada na correção das manifestações patológicas, uma vez que o padrão observado indica que o problema não é solucionado quando as suas causas não são eliminadas. Ao contrário disso, a estrutura tornase vulnerável ao aparecimento de novas anomalias ou ao agravamento das já existentes.

A Tabela 2 apresenta a divisão de manifestações patológicas encontradas, além dos valores dos parâmetros GUT adotados, bem como a pontuação final e o número de casos incidentes em cada categoria.

Tabela 2: Valores propostos para os parâmetros GUT das principais anomalias encontradas.

\begin{tabular}{|c|c|c|c|c|c|c|c|}
\hline \multicolumn{2}{|c|}{ Manifestação Patológica } & \multirow{2}{*}{ Distinção } & \multirow{2}{*}{ G } & \multirow{2}{*}{$\mathbf{u}$} & \multirow{2}{*}{$\mathbf{T}$} & \multirow{2}{*}{ GUT } & \multirow{2}{*}{ Incidência } \\
\hline Grupo & Subgrupo & & & & & & \\
\hline \multirow{2}{*}{ Corrosão de Armadura } & I & Casos pouco pronunciados em elementos estruturais & 3 & 2 & 3 & 18 & 59 \\
\hline & II & Casos avançados em elementos estruturais muito solicitados & 4 & 3 & 3 & 36 & 6 \\
\hline \multirow{2}{*}{ Fissuras } & I & Fissuras & 2 & 1 & 2 & 4 & 55 \\
\hline & II & Trincas & 2 & 2 & 3 & 12 & 29 \\
\hline Eflorescência & - & - & 2 & 2 & 2 & 8 & 19 \\
\hline Segregação & - & - & 2 & 2 & 1 & 4 & 4 \\
\hline \multirow[b]{2}{*}{ Desagregação } & I & Pequena perda de massa em elementos estruturais & 2 & 2 & 3 & 12 & 60 \\
\hline & II & $\begin{array}{l}\text { Grande perda de massa em elementos estruturais muito } \\
\text { solicitados }\end{array}$ & 2 & 3 & 3 & 18 & 47 \\
\hline Manchas & - & - & 2 & 2 & 1 & 4 & 16 \\
\hline
\end{tabular}

Fonte: Autores (2017).

Através da Figura 4 é possível observar como foi 145 distribuída a incidência das manifestações patológicas de uma forma geral. Observa-se a http: / /dx.doi.org/10.25286/repa.v2i3.707 
predominância de desagregação, fissuras e corrosão de armadura.

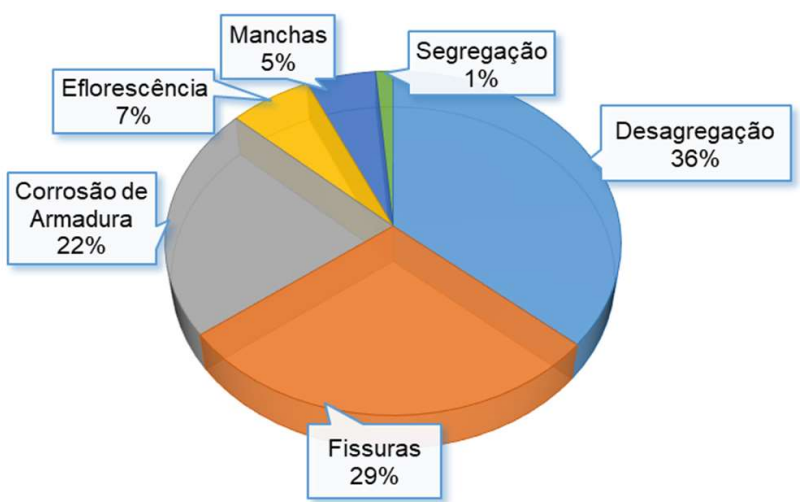

Figura 4: Manifestações patológicas em concreto armado no Centro de Tecnologia da UFPB.

Fonte: Autores (2017).

A Tabela 3 traz a lista com a priorização obtida através da implementação do método GUT. De acordo com a Tabela 3, em uma situação de recursos financeiros limitados, a aplicação de correção às manifestações patológicas encontradas nas edificações da área de estudo deve priorizar os elementos estruturais que apresentam um quadro de corrosão de armadura agravado (II).

Tabela 3: Priorização.

\begin{tabular}{c|c}
\hline Categoria & Prioridade \\
\hline Corrosão de Armadura II & 1 \\
\hline Corrosão de Armadura I & 2 \\
\hline Desagregação II & 2 \\
\hline Desagregação I & 3 \\
\hline Fissura II & 3 \\
\hline Eflorescência & 4 \\
\hline Fissura I & 5 \\
\hline Manchas & 5 \\
\hline Segregação & 5
\end{tabular}

Fonte: Autores (2017).

\section{Conclusões}

Com base no estudo realizado e resultados obtidos, observa-se a presença de um elevado número de anomalias em concreto armado na área de estudo. Entre elas, destaca-se a incidência de fissuras, desagregação do concreto e corrosão de armadura. Além disso, a priorização por meio do método GUT sugere que, no local estudado, os recursos destinados à manutenção sejam aplicados para realizar correções nos elementos estruturais que possuem armaduras em estado avançado de corrosão, seguidos daqueles com grau mais leve de comprometimento.

A priorização realizada dessa forma guia a realização de manutenção conjunta em todas as edificações visitadas, de acordo com as manifestações patológicas localizadas no topo da lista.

Conclui-se que a ferramenta GUT pode ser aplicada como facilitadora do planejamento das atividades de manutenção em edificações de concreto armado, com base nos resultados e na avaliação proposta.

O emprego do método GUT pode ser justificado pela busca por oportunidade de melhorias nas etapas de projeto, execução e manutenção de estruturas de concreto armado, através da retroalimentação do sistema, possibilitada pela análise das prováveis origens das manifestações patológicas. Sugere-se, em trabalhos futuros:

- Realizar o mapeamento que aponte as prováveis fases nas quais as manifestações patológicas tiveram origem, produzindo informações necessárias à retroalimentação do processo;

- Avaliar a aplicabilidade do método em empresas do ramo da construção civil e instituições públicas na busca por otimização na elaboração de projetos e na execução de obras, através da implantação de um processo de retroalimentação;

- Realizar ensaios detalhados de inspeção (carbonatação, esclerometria, termografia infravermelha etc.), uma vez que podem contribuir para a obtenção de um diagnóstico mais acurado. 


\section{Referências}

[1] THOMAZ, E. Trincas em edifícios: causas, prevenção e recuperação. São Paulo, Ed. PINI, 1992.

[2] MEIRA, G. R. Corrosão de Armaduras em estruturas de concreto: fundamentos, diagnóstico e prevenção. IFPB. João Pessoa, 2017.

[3] GUIMARÃES L. E.; CARASEK, H. ; CASCUDO, O. Proposta de metodologia para avaliação comparativa do grau de deterioração em edificações. In: CONPAT. Mérida, Yucatán, México, 2003.

[4] ANDRADE, T.; SILVA, A.J.C. Patologia das Estruturas. In: ISAIA, Geraldo Cechella. (Ed) Concreto: In.: Concreto: Ensino, Pesquisa e Realizações. Editor: Geraldo Cechella Isaia. São Paulo: IBRACON, 2005, V.1, Cap. 32.

[5] SILVA, L. k. Levantamento de Manifestações Patológicas em Estruturas de Concreto Armado no Estado do Ceará. Monografia. Universidade Federal do Ceará, Ceará, 2011.

[6] SOUZA, V. C.; RIPPER, T. Patologia, recuperação e reforço de estruturas de concreto. São Paulo: Pini, 1998

[7] BRANDÃO, A.M.S. Qualidade e durabilidade das estruturas de concreto armado: aspectos relativos ao projeto. Dissertação (Mestrado), Escola de Engenharia de São Carlos - Universidade de São Paulo, São Carlos, 1998.

[8] FIGUEIREDO, A.D. Concreto com Fibras. In.: Concreto: Ensino, Pesquisa e Realizações. Editor: Geraldo Cechella Isaia. São Paulo: IBRACON, 2005, V.2, Cap. 39.

[9] MOLIN, D.C.C.D.; PAULETTI, C.; POSSAN, E. Carbonatação acelerada: Estado da arte das pesquisas no Brasil. Ambiente construído, Porto Alegre, v.7, no 4, p.7-20, 2007.
[10] ASSOCIAÇÃO BRASILEIRA DE NORMAS TÉCNICAS. NBR 6118: Projeto de Estruturas de Concreto: Procedimento. Rio de Janeiro, 2014.

[11] ARANHA, P.M.S. Contribuição ao estudo das manifestações patológicas em estruturas de concreto armado na região amazônica. Dissertação (Mestrado), Universidade Federal do Rio Grande do Sul, Porto Alegre, 1994.

[12] ANDRADE, J.J.O. Durabilidade das estruturas de concreto armado: análise das manifestações patológicas nas estruturas no estado de Pernambuco. Dissertação (Mestrado), Universidade Federal do Rio Grande do Sul, Porto Alegre, 1997.

[13] NINCE, A.A. Levantamento de dados sobre causas de deterioração de estruturas na região Centro-Oeste. Dissertação (Mestrado), Universidade de Brasília, Brasília, 1996.

[14] LICHTENSTEIN, N. B. Patologia das construções: procedimentos para formulação do diagnóstico de falhas e definição de conduta adequada à recuperação de edificações. São Paulo. 191p. Dissertação (Mestrado) - Escola Politécnica da Universidade de São Paulo, 1985.

[15] GNIPPER, S. F. Diretrizes para formulação de método hierarquizado para investigação de patologias em sistemas prediais hidráulicos e sanitários. Dissertação (Mestrado). Universidade Estadual de Campinas, Faculdade de Engenharia Civil, Arquitetura e Urbanismo, Campinas, São Paulo, 2010.

[16] PACHECO, C.; VIEIRA, G. Metodologias para inspeções de fachadas de edifícios In: IV Congresso Internacional na "Recuperação, Manutenção e Restauração de Edifícios" CIRMARE. Anais. Rio de Janeiro, 2015.

[17] SILVA, S. V.; MONTEIRO, E. C. B. Aplicação das ferramentas da qualidade FMEA e FTA para detecção e controle de patologias em estruturas de concreto armado do Aeroporto Internacional do Recife. Revista de Engenharia e Pesquisa Aplicada, v.1, no1, p.3-12, 2016.

http: / /dx.doi.org/10.25286/repa.v2i3.707 
[18] INSTITUTO BRASILEIRO DE AVALIAÇÕES E PERÍCIAS DE ENGENHARIA (IBAPE). Norma de Inspeção Predial Nacional. São Paulo, 2012.

[19] KEPNER, Charles H.; TREGOE, Benjamin B. O administrador racional. São Paulo: Atlas, 1981

[20] OLIVEIRA, Sidney T. Ferramentas para o aprimoramento da qualidade. Série Qualidade Brasil. 1995.

[21] KNAPP, L. M.; OLIVAN, L. I. Classificação de Desempenho de edificação Habitacional Método GUT - Estruturas Metálicas. In: XVIII COBREAP Congresso Brasileiro de Engenharia de Avaliação e Perícias, Belo Horizonte, 2015. 